\title{
Cambios en el agua corporal, grasa y masa magra de personas con y sin disfunción renal en sus diversos estadios de enfermedad renal crónica
}

Changes in body water, lean body mass and body fat in persons with and without renal disfunction at several stages of chronic renal disease

Ana Rosa Vilela Sangay ${ }^{1, \mathrm{c}}$ Javier Antonio Cieza Zevallos ${ }^{1, a ;}$, 2,b, Celene Uriol Lescano $^{1, \mathrm{c}}$

\section{RESUMEN}

Objetivo: Estudiar los cambios en el agua corporal, grasa y masa magra de personas sanas y enfermas (20-80 años) categorizadas según tasa de filtración glomerular (TFG). Material y métodos: Estudio descriptivo y analítico de 198 sujetos (sanos, con enfermedades crónicas sin azoemia y con diferentes estados de enfermedad renal crónica normalizados por sexo) según categorías: Categoría 1 (TFG $>140 \mathrm{ml} / \mathrm{min} / 1,73 \mathrm{~m}^{2} \mathrm{SC}$ ); Categoría 2 (TFG 90-140); Categoría 3 (TFG 60-89); Categoría 4 (TFG 30-59); Categoría 5 (TFG<30) y Categoría 6 (diálisis). Se midió peso, talla, agua corporal, grasa y masa magra con balanza de bioimpedancia; aclaramiento de creatinina (TFG), aclaramiento de urea y sodio y potasio en orina de 24 horas. Con estos datos se calculó la masa corporal, agua corporal, grasa y masa magra, la ingesta de sal sódica, potasio y proteínas. Se relacionaron las diversas variables según categorías propuestas con ANOVA y eta cuadrado. Se correlacionó con la TFG y la masa magra mediante regresión bivariada y el resto de variables mediante regresión múltiple lineal, para definir las relevantes. Resultados: Las variables relevantes asociadas con pérdida de TFG fueron la masa magra y el agua corporal: ANOVA ( $\mathrm{p}=0,000$ ambas) y eta cuadrado $(0,178$ y 0,165$)$, respectivamente. El análisis multivariado solo relacionó la TFG con la edad $(\mathrm{r}=-0,34 ; \mathrm{p}=0,000)$ y la masa magra mostró correlación bivariada relevante con el agua $(\mathrm{r}=0,861 ; \mathrm{p}=0,000)$. Conclusión: La pérdida de TFG implica fundamentalmente pérdida de masa magra y agua corporal en la estructura corporal.

PALABRAS CLAVE: Agua corporal, grasa corporal, masa magra, función renal. (Fuente: DeCS BIREME).

\section{SUMMARY}

Objective: To study changes in body water, fat and lean body mass in healthy and sick persons (20-80 years of age) by glomerular filtration rate (GFR). Methods: A descriptive study was conducted among 198 subjects (healthy, with chronic renal disease without azotemia and with several stages of chronic renal disease by sex) using the following categories: Category 1 (GFR >140 ml/min/1.73 m²SC); Category 2 (GFR 90-140); Category 3 (GFR 60-89); Category 4 (GFR 30-59); Category 5 (GFR $<30$ ) and Category 6 (dialysis). Body weight, height, body water, body fat and lean body mass were measured using bioimpedance. Creatinine clearance (GFR), urea clearance, sodium

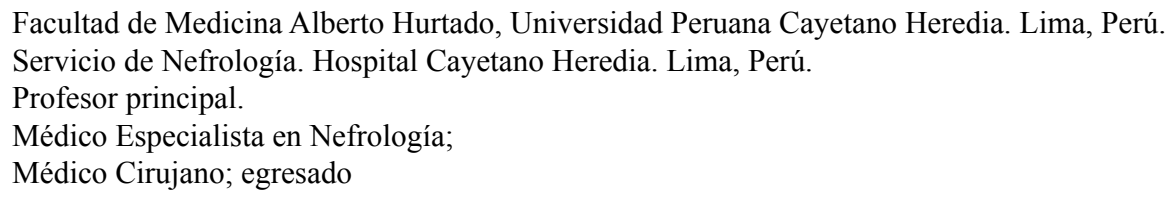


and potassium were measured in 24-hour urine collection. ANOVA and eta square were used to contrast variables according to the proposed categories. GFR and lean body mass were correlated using bivariate regression and the rest of the variables with linear regression. Results: Relevant variables associated with loss of GFR were lean body mass and body water: ANOVA ( $\mathrm{p}=0,000$ for both) and eta square $(0,178$ y 0,165$)$, respectively. Multivariate analysis only correlated GFR with age $(\mathrm{r}=-0,34 ; \mathrm{p}=0,000)$ and lean body mass showed correlation with body water $(\mathrm{r}=0,861$; $\mathrm{p}=0,000$. Conclusions: Loss of GFR implies fundamentally loss of lean body mass and body water.

KEYWORDS: Body water, body fat, lean mass, renal function. (Source: MeSH NLM.)

\section{INTRODUCCIÓN}

Nuestro cuerpo como materia biológica debe seguir un proceso que, inicia en una génesis sostenible seguido de una evolución, culmina en la madurez e implica su plenitud funcional que lo torna armónico con su entorno ambiental, y posteriormente, ocurre un decrecimiento que finaliza con la muerte de esta materia biológica.

Como todo proceso, esta materia biológica en el ser humano (el cuerpo), está sujeta a interferencias que pueden distorsionarla y cambiar el curso esperable para mejorarlo o deteriorarlo. Cuando el proceso sucede armónicamente, el individuo alcanza su plenitud o madurez biológica en los tiempos previstos y mantiene un equilibrio estructural que, a su vez, le permite una existencia acorde con su medio ambiente, situación que generalmente llamamos salud.

Alcanzada esta madurez, mantener la armonía de los grandes componentes corporales permite el desarrollo de otra índole de actividades, ya no necesariamente materiales, sino, intelectuales, psicológicos y del saber filosófico. Finalmente, la declinación del proceso de madurez biológica conlleva el término de la vida material, pero en muchas situaciones, esta etapa se acompaña de la sabiduría de la persona y el esplendor que refleja casi siempre un proceso biológico que ha transcurrido armoniosamente.

Para que este proceso ocurra saludablemente es indispensable que el "medio interno" se mantenga estable para que las unidades biológicas (las células), aun cuando en masa estuvieran disminuidas, cumplan sus funciones a cabalidad y por ello, uno de los sistemas fundamentales para cumplir este objetivo, es el sistema renal que lo expresaremos como el aclaramiento de la creatinina.

Al respecto, un estudio realizado en personas sanas de la ciudad de Lima mostró que, esta hipótesis de reestructuración armoniosa del cuerpo humano, efectivamente así ocurre y consistentemente la depuración de creatinina cambia también con la edad, en un modelo de regresión cúbica con pendiente negativa tanto en hombres como mujeres entre los veinte y los ochenta años de edad, pero donde el valor de la creatinina y la urea séricas se mantienen constantes (1). Esto muestra con toda claridad la existencia de sistemas de autocontrol en la vida saludable de la persona sana que le permiten una existencia corporal satisfactoria a cabalidad, por lo menos hasta los ochenta años.

Esta observación conlleva la pregunta: ¿qué ocurre cuando por cualquier causa hay pérdida de esta armonía expresada a través de la disminución de la función renal?, ¿se pierde este sistema de regulación de las grandes estructuras de nuestro cuerpo?

$\mathrm{Al}$ respecto, hay trabajos en la literatura internacional que muestran por ejemplo cambios del peso corporal, pero sin mucha precisión $(2,3)$. Además, como cada población o individuo estructura su cuerpo en formas diversas, es importante hacer conciencia de estos cambios en poblaciones como las nuestras antropométricamente diferentes, con una cultura, hábitos alimentarios distintos y entornos o medio ambientes diversos.

Este estudio tuvo como objetivo principal estudiar los cambios en el agua corporal, grasa y masa magra de sujetos sanos y/o enfermos entre 20 y 80 años de edad, pero con diferentes estados de función renal, evaluados mediante el aclaramiento de creatinina medido y apareados en función del sexo.

\section{MATERIAL Y MÉTODOS}

Estudio observacional de 198 casos realizado entre el 15 enero del 2018 y 15 marzo del 2019 en personas con y sin disminución de la tasa de filtración glomerular (TFG) medido mediante el aclaramiento 
de creatinina en 24 horas $(\mathrm{Cl} \mathrm{Cr})$ y agrupados en las siguientes categorías:

Categoría 1: sujetos con TFG o $\mathrm{Cl} \mathrm{Cr}>140 \mathrm{ml} /$ $\mathrm{min} / 1,73 \mathrm{~m}^{2} \mathrm{SC}$;

Categoría 2: sujetos con TFG o $\mathrm{Cl}$ Cr entre 90 y 140 $\mathrm{ml} / \mathrm{min} / 1,73 \mathrm{~m}^{2} \mathrm{SC}$;

Categoría 3: sujetos con TFG o $\mathrm{Cl}$ CR entre 60 y 89 $\mathrm{ml} / \mathrm{min} / 1,73 \mathrm{~m}^{2} \mathrm{SC}$;

Categoría 4: sujetos con TFG o Cl Cr entre 30 y $59 \mathrm{ml} /$ $\min / 1,73 \mathrm{~m}^{2} \mathrm{SC}$;

Categoría 5: sujetos con TFG o $\mathrm{Cl} \mathrm{Cr}<30 \mathrm{ml} / \mathrm{min} / 1,73$ $\mathrm{m}^{2} \mathrm{SC}$ y

Categoría 6: sujetos en terapia dialítica.

Los sujetos fueron tanto personas sanas como enfermas que adolecían de enfermedades crónicas pero que se encontraban hemodinámicamente estables y con motricidad conservada, incluidos por incidencia accidental al Hospital Cayetano Heredia entre enero del 2018 y marzo del 2019. Se seleccionó para cada categoría en lo posible un hombre por una mujer y de edades similares.

Se consideró persona sana, aquella que no adolecía o ignoraba tener alguna enfermedad, nunca se había hospitalizados, no tomaba medicamento alguno y consideraba tener una vida plena en sus actividades cotidianas. Se excluyeron personas menores de 18 años, gestantes y enfermos que tuvieran edema o requerían tratamientos de urgencia médica, tuvieran un IMC mayor de 30, o que usaban diuréticos. La participación fue voluntaria.

A todos los participantes se les midió el peso, talla, agua corporal, grasa y masa magra con una balanza con medición de bioimpedancia. Se determinó el aclaramiento de creatinina y urea con medición en sangre y orina de urea y creatinina. La orina fue recolectada en 24 horas con excepción de las personas en diálisis. Se calculó el índice de masa corporal (IMC), el agua en litros y la grasa y masa magra en kilogramos ajustado al peso corporal de cada persona (porcentaje/peso de la persona). Se calculó la excreción urinaria diaria de creatinina y de urea por $\mathrm{kg}$ de peso corporal de cada persona. Asimismo, se midió la excreción diaria de sodio, potasio y cloro en la orina de 24 horas, con lo que se calculó la ingesta asumida de sal sódica, potasio y proteínas diarias.

Se usó estadística descriptiva al inicio, para describir las características generales de los casos estudiados, y posteriormente, se realizó comparación de las medias de las diversas variables estudiadas en función de las categorías pre establecido, usando el test de ANOVA y eta cuadrado que permite una mejor interpretación de su significancia. También se analizaron las principales correlaciones de las variables bajo observación con el $\mathrm{Cl} \mathrm{Cr}$ mediante regresión bivariada, y en aquellas que se consideró necesario, se realizó regresión múltiple lineal para definir las variables que influencian la variable bajo observación. Se aceptó como significancia estadística un $\mathrm{p}<0,05$.

El estudio fue revisado y aprobado por el Comité de Ética del Hospital Cayetano Heredia de Lima Perú.

\section{RESULTADOS}

Se estudiaron 198 personas (99 hombres y 99 mujeres) distribuidos según sexo en cada categoría en forma probabilísticamente equitativa $(\mathrm{p}=0,973)$. La edad media de los hombres estudiados fue 50,7 $\pm 21,2$ años y de las mujeres fue $49,5 \pm 18,7$ años $(\mathrm{p}=0,666)$. En la tabla 1 se muestran las características de las

Tabla 1. Características de los sujetos según categorías de tasa de filtración glomerular.

\begin{tabular}{|c|c|c|c|c|c|c|c|c|}
\hline & \multicolumn{6}{|c|}{ Aclaramiento creatinina $\left(\mathrm{ml} / \mathrm{min} / 1,73 \mathrm{~m}^{2} \mathrm{SC}\right)$} & \multicolumn{2}{|c|}{$\mathbf{p}$} \\
\hline & $\begin{array}{c}>140 \\
(n=26)\end{array}$ & $\begin{array}{c}\text { Entre 140-90 } \\
\quad(n=38)\end{array}$ & $\begin{array}{c}\text { Entre 90-60 } \\
\qquad(n=28)\end{array}$ & $\begin{array}{c}\text { Entre 60-30 } \\
\quad(n=21)\end{array}$ & $\begin{array}{c}<30 \\
(n=39)\end{array}$ & $\begin{array}{l}\text { En diálisis } \\
(\mathrm{n}=46)\end{array}$ & Anova & $\begin{array}{c}\text { eta } \\
\text { cuadrado }\end{array}$ \\
\hline Edad (años) & $34,7 \pm 13,1$ & $43,4 \pm 19,8$ & $55,5 \pm 22,1$ & $58,2 \pm 21,0$ & $62,0 \pm 14,7$ & $47,4 \pm 17,5$ & 0,000 & 0,203 \\
\hline Talla (m) & $1,64 \pm 0,08$ & $1,63 \pm 0,09$ & $1,62 \pm 0,10$ & $1,61 \pm 0,10$ & $1,54 \pm 0,09$ & $1,55 \pm 0,09$ & 0,000 & 0,168 \\
\hline Peso (kg) & $63.3 \pm 8,71$ & $62,9 \pm 9,51$ & $64,9 \pm 9,15$ & $64,0 \pm 10,3$ & $58,0 \pm 9,6$ & $53,9 \pm 11,6$ & 0,000 & 0,154 \\
\hline IMC $\left(\mathrm{kg} / \mathrm{m}^{2}\right)$ & $23,5 \pm 2,32$ & $23,7 \pm 2,68$ & $24,7 \pm 2,84$ & $24,7 \pm 3,24$ & $24,4 \pm 3,74$ & $22,5 \pm 4,94$ & 0,075 & 0,050 \\
\hline Hombres (\%) & $46 \%$ & $47 \%$ & $54 \%$ & $57 \%$ & $49 \%$ & $50 \%$ & 0,957 & \\
\hline
\end{tabular}

Media \pm DS 
Tabla 2. Tipo de persona estudiada según categorías de la tasa de filtración glomerular.

\begin{tabular}{|c|c|c|c|c|c|c|c|}
\hline \multirow[b]{2}{*}{ Tipo de persona } & \multicolumn{6}{|c|}{ Aclaramiento de creatinina $\left(\mathrm{ml} / \mathrm{min} / 1,73 \mathrm{~m}^{2} \mathrm{SC}\right) \mathrm{n}(\%)$} & \multirow[b]{2}{*}{ Total } \\
\hline & $>140$ & $140-90$ & $90-60$ & $60-30$ & $<\mathbf{3 0}$ & $\begin{array}{c}\text { En } \\
\text { Diálisis }\end{array}$ & \\
\hline Sanos & $18(69 \%)$ & $22(58 \%)$ & $11(39 \%)$ & 0 & 0 & 0 & $51(26 \%)$ \\
\hline Enfermos crónicos asintomáticos & $8(31 \%)$ & $16(42 \%)$ & $11(39 \%)$ & $9(43 \%)$ & $2(5 \%)$ & 0 & $46(23 \%)$ \\
\hline Enfermos crónicos sintomáticos leves & 0 & 0 & $6(21 \%)$ & $12(57 \%)$ & $11(28 \%)$ & 0 & $29(15 \%)$ \\
\hline Enfermos crónicos sintomáticos moderados & 0 & 0 & 0 & 0 & $26(67 \%)$ & 0 & $26(13 \%)$ \\
\hline En diálisis & 0 & 0 & 0 & 0 & 0 & $\begin{array}{c}46 \\
(100 \%)\end{array}$ & $46(23 \%)$ \\
\hline Total & 26 & 38 & 28 & 21 & 39 & 46 & 198 \\
\hline
\end{tabular}

Tabla 3. Características estructurales según categorías de tasa de filtración glomerular.

\begin{tabular}{|c|c|c|c|c|c|c|c|c|c|}
\hline & \multicolumn{7}{|c|}{ Aclaramiento de creatinina $\left(\mathrm{ml} / \mathrm{min} / 1,73 \mathrm{~m}^{2} \mathrm{SC}\right)$} & \multicolumn{2}{|c|}{$\mathbf{p}$} \\
\hline & $\begin{array}{c}>140 \\
(n=26)\end{array}$ & $\begin{array}{l}140-90 \\
(n=38)\end{array}$ & $\begin{array}{c}90-60 \\
(n=28)\end{array}$ & $\begin{array}{c}60-30 \\
(n=21)\end{array}$ & $\begin{array}{c}<30 \\
(n=39)\end{array}$ & $\begin{array}{c}\text { En } \\
\text { Diálisis } \\
(n=46)\end{array}$ & $\begin{array}{c}\text { Total } \\
(n=198)\end{array}$ & ANOVA & $\begin{array}{c}\text { eta } \\
\text { cuadrado }\end{array}$ \\
\hline Agua (\%) & $53,2 \pm 4,4$ & $52,0 \pm 5,9$ & $50,7 \pm 5,4$ & $52,3 \pm 4,0$ & $49,2 \pm 4,9$ & $52,3 \pm 5,5$ & $51,7 \pm 5,3$ & 0,011 & 0,074 \\
\hline Agua (l) & $33,7 \pm 5,3$ & $32,7 \pm 5,6$ & $33,0 \pm 6,4$ & $33,5 \pm 5,7$ & $28,4 \pm 4,9$ & $28,3 \pm 5,3$ & $31,1 \pm 5,9$ & 0,000 & 0,165 \\
\hline Grasa $(\%)$ & $26,7 \pm 5,6$ & $28,8 \pm 8,1$ & $30,5 \pm 7,4$ & $28,4 \pm 6,7$ & $34,0 \pm 6,6$ & $28,1 \pm 7,7$ & $29,4 \pm 7,5$ & 0,004 & 0,099 \\
\hline Grasa $(\mathbf{k g})$ & $17,0 \pm 4,7$ & $18,3 \pm 6,1$ & $14,4 \pm 9,2$ & $9,4 \pm 9,7$ & $14,9 \pm 9,5$ & $15,5 \pm 6,0$ & $15,3 \pm 7,9$ & 0,002 & 0,095 \\
\hline $\begin{array}{l}\text { Masa magra } \\
(\%)\end{array}$ & $38,7 \pm 4,9$ & $37,4 \pm 6,7$ & $33,5 \pm 7,3$ & $35,9 \pm 7,3$ & $33,1 \pm 6,8$ & $37,5 \pm 7,6$ & $36,3 \pm 7,1$ & 0,010 & 0,086 \\
\hline $\begin{array}{l}\text { Masa magra } \\
(\mathrm{kg})\end{array}$ & $46,4 \pm 7,0$ & $42,5 \pm 8,9$ & $\begin{array}{c}45,1 \pm \\
10,1\end{array}$ & $43,4 \pm 11,4$ & $35,6 \pm 5,9$ & $38,5 \pm 7,7$ & $41,2 \pm 8,9$ & 0,000 & 0,178 \\
\hline
\end{tabular}

Media \pm DS

Tabla 4. Correlación lineal bivariada entre las características estructurales de los sujetos estudiados y la TFG $(n=214)$.

\begin{tabular}{lccc}
\hline Aclaramiento de creatinina $\left.\mathbf{( m l} / \mathbf{m i n} / \mathbf{1 , 7 3} \mathbf{~ m}^{\mathbf{2}} \mathbf{~ S C}\right)$ & $\mathbf{r}$ Pearson & $\mathbf{p}$ & $\mathbf{n}$ \\
\hline Edad (años) & $-0,346$ & 0,000 & 196 \\
Talla (m) & 0,375 & 0,000 & 196 \\
Peso (kg) & 0,275 & 0,000 & 196 \\
Agua (\%) & 0,108 & 0,132 & 196 \\
Agua (l) & 0,335 & 0,000 & 196 \\
Grasa (\%) & $-0,160$ & 0,038 & 170 \\
Grasa (kg) & 0,136 & 0,058 & 196 \\
Masa magra (\%) & 0,145 & 0,059 & 170 \\
Masa magra (kg) & 0,352 & 0,000 & 170 \\
\hline
\end{tabular}

categorías definidas en material y método en función de la edad, talla, peso, IMC y sexo. El valor promedio de la TFG $\left(\mathrm{ml} / \mathrm{min} / 1,73 \mathrm{~m}^{2} \mathrm{SC}\right)$ medido mediante el $\mathrm{Cl}$ Cr de cada categoría fue: Categoría 1: $176 \pm 29,69$ $(\mathrm{n}=26)$; Categoría 2: $110 \pm 13,54(\mathrm{n}=38)$; Categoría 3: $75 \pm 8,41(\mathrm{n}=28)$; Categoría 4: $45 \pm 8,53 \quad(\mathrm{n}=21)$; Categoría 5: $12 \pm 6,36(n=39)$ y Categoría 6: $0(n=46)$ $\mathrm{ml} / \mathrm{min} / 1,73 \mathrm{~m}^{2} \mathrm{SC}$. 
Tabla 5. Correlación lineal bivariada entre las características estructurales de los sujetos estudiados y la TFG $(n=100)$.

\begin{tabular}{lccr}
\hline Aclaramiento creatinina (ml/min/1,73 $\left.\mathbf{m}^{\mathbf{2}} \mathbf{S C}\right)$ & $\mathbf{r}$ Pearson & $\mathbf{p}$ & $\mathbf{n}$ \\
\hline Urea sérica (mg/dl) & $-0,471$ & 0,000 & 100 \\
Urea orina $\mathbf{2 4}$ horas (g/d) & 0,599 & 0,000 & 100 \\
Consumo sal sódica/día (g) & 0,366 & 0,000 & 99 \\
Consumo de potasio (mEq/kg) & 0,512 & 0,000 & 99 \\
Ingesta proteínas (g/kg/día) & 0,632 & 0,000 & 100 \\
\hline
\end{tabular}

Tabla 6. Correlación lineal bivariada entre la masa magra y variables estudiadas con significancia estadística.

\begin{tabular}{lccc}
\hline \multicolumn{1}{c}{ Masa Magra (kg) } & r Pearson & p & n \\
\hline Agua (litros) & 0,861 & 0,000 & 172 \\
Grasa (\% peso) & $-0,331$ & 0,000 & 172 \\
Creatinina sérica (mg/dl) & $-0,263$ & 0,000 & 172 \\
Creatinina orina (mg/kg/día) & 0,346 & 0,000 & 100 \\
Urea sérica (mg/dl) & $-0,277$ & 0,001 & 172 \\
Urea orina diaria (g) & 0,336 & 0,001 & 172 \\
\hline
\end{tabular}

Tabla 7. Regresión lineal multivariada de la tasa de filtración glomerular y las variables estructurales de las personas y la edad.

\begin{tabular}{|c|c|c|c|c|}
\hline \multirow{2}{*}{$\begin{array}{l}\text { Variable de estudio: TFG }(\mathrm{ml} / \\
\left.\qquad \mathrm{min} / \mathrm{m}^{2} \mathrm{SC}\right)\end{array}$} & \multicolumn{2}{|c|}{$\begin{array}{l}\text { Coeficientes no } \\
\text { estandarizados }\end{array}$} & \multirow{2}{*}{$\begin{array}{c}\begin{array}{c}\text { Coeficientes } \\
\text { tipificados }\end{array} \\
\text { Beta }\end{array}$} & \multirow{2}{*}{$p$} \\
\hline & $\boldsymbol{B}$ & $\mathbf{E E}$ & & \\
\hline (Constante del modelo) & 122 & 541 & & 0.822 \\
\hline Agua (litros) & -1.89 & 3.47 & -0.18 & 0.587 \\
\hline Masa magra (kg) & -0.19 & 1.07 & -0.03 & 0.856 \\
\hline Grasa (kg) & 0.26 & 2.65 & 0.02 & 0.921 \\
\hline Edad (años) & -1.12 & 0.27 & -0.34 & 0 \\
\hline Talla (m) & -32.17 & 341.6 & -0.05 & 0.925 \\
\hline Peso (kg) & 4.86 & 5.54 & 0.8 & 0.381 \\
\hline IMC $\left(\mathrm{kg} / \mathrm{m}^{2}\right)$ & -7.6 & 11.26 & -0.41 & 0.501 \\
\hline
\end{tabular}

Las características clínicas del tipo de persona estudiada en función de la TFG se presentan en la tabla 2. En las tablas 3 y 4 se presenta las características de sus estructuras corporales en función de la TFG y en la tabla 4 las correlaciones existentes entre éstas y al aclaramiento de creatinina medido.

En la tabla 5 se presenta la correlación bivariada entre la TFG y la urea sérica y la excretada en orina de 24 horas así como el consumo de sal sódica diaria y de potasio y proteínas ajustadas estas dos últimas al peso corporal y extrapoladas de la medida de sodio, potasio y urea en orina de 24 horas, habiéndose excluido los pacientes que consumían diuréticos.

Considerando el resultado de los análisis de ANOVA y eta cuadrados presentados en la tabla 3, se muestran los gráficos 1 y 2: masa magra ajustada a peso de la persona (en $\mathrm{kg}$ ) y agua (litros) que objetiva los valores eta cuadrado $>0,14$. Complementariamente, se presenta en el gráfico 3 los cambios observados en la grasa corporal ajustada al peso de la persona, reconociendo que el valor del eta cuadrado de esta variable es de efecto moderado (eta 0,095). 


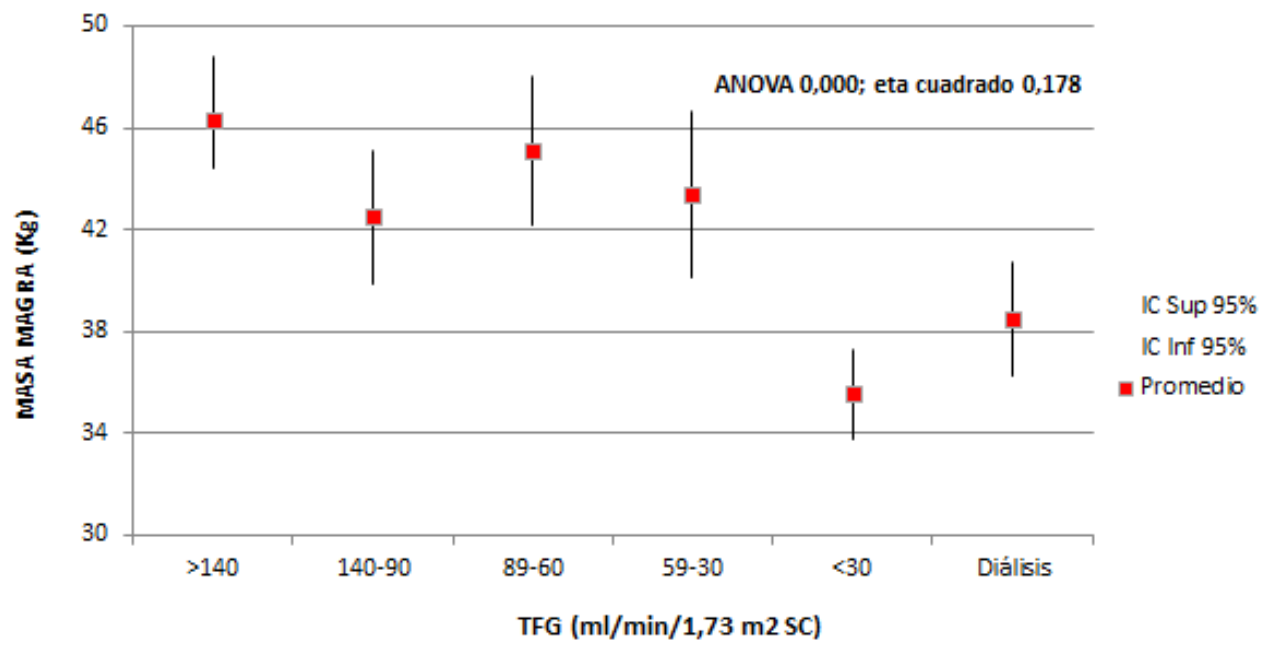

Gráfico 1. Relación entre la masa magra y el aclaramiento de creatinina categorizado de los sujetos estudiados.

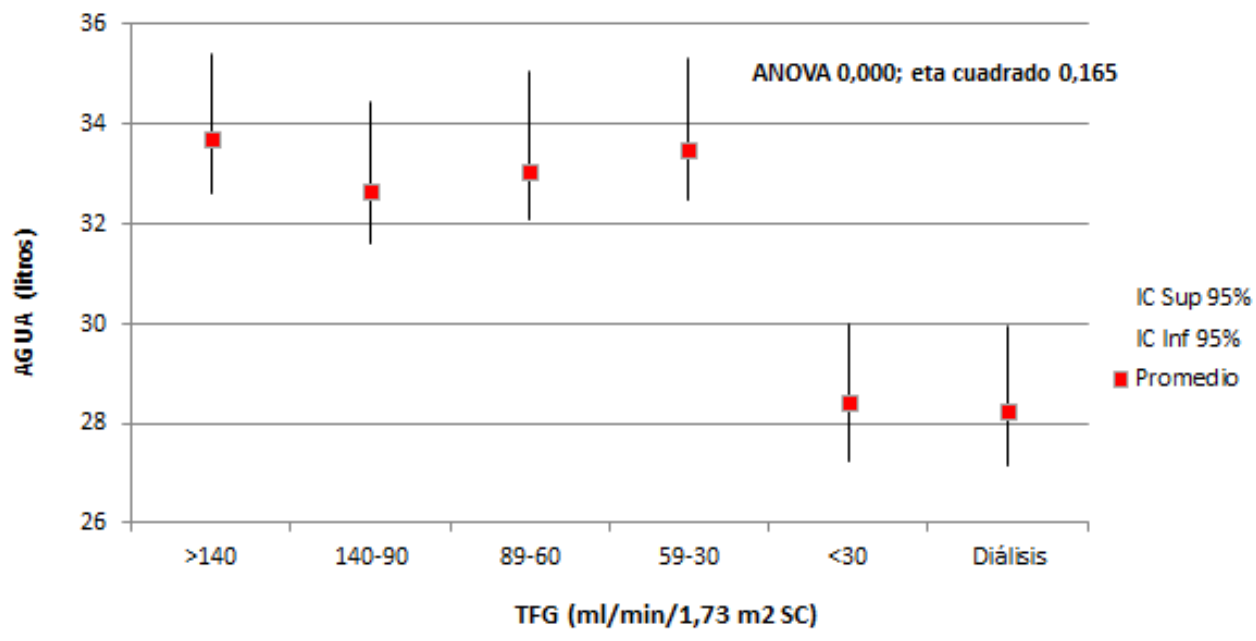

Gráfico 2. Relación entre el agua corporal y el aclaramiento de creatinina categorizado de los sujetos estudiados.

Siendo la masa magra ajustada al peso de la persona una variable relevante, se analizó su correlación lineal bivariada (tabla 6) y multivariada que mostró que únicamente el agua corporal en litros tenía una muy buena correlación (gráfico 4). Ninguna de las otras variables mostró significancia en el análisis multivariado.
El análisis lineal multivariado del aclaramiento de creatinina, considerándola como variable numérica continua, mostró que la única variable relacionada fue la edad, aun cuando en la tabla 4 se puede observar que hubo otras variables con correlación estadísticamente significativa en el análisis bivariado. 


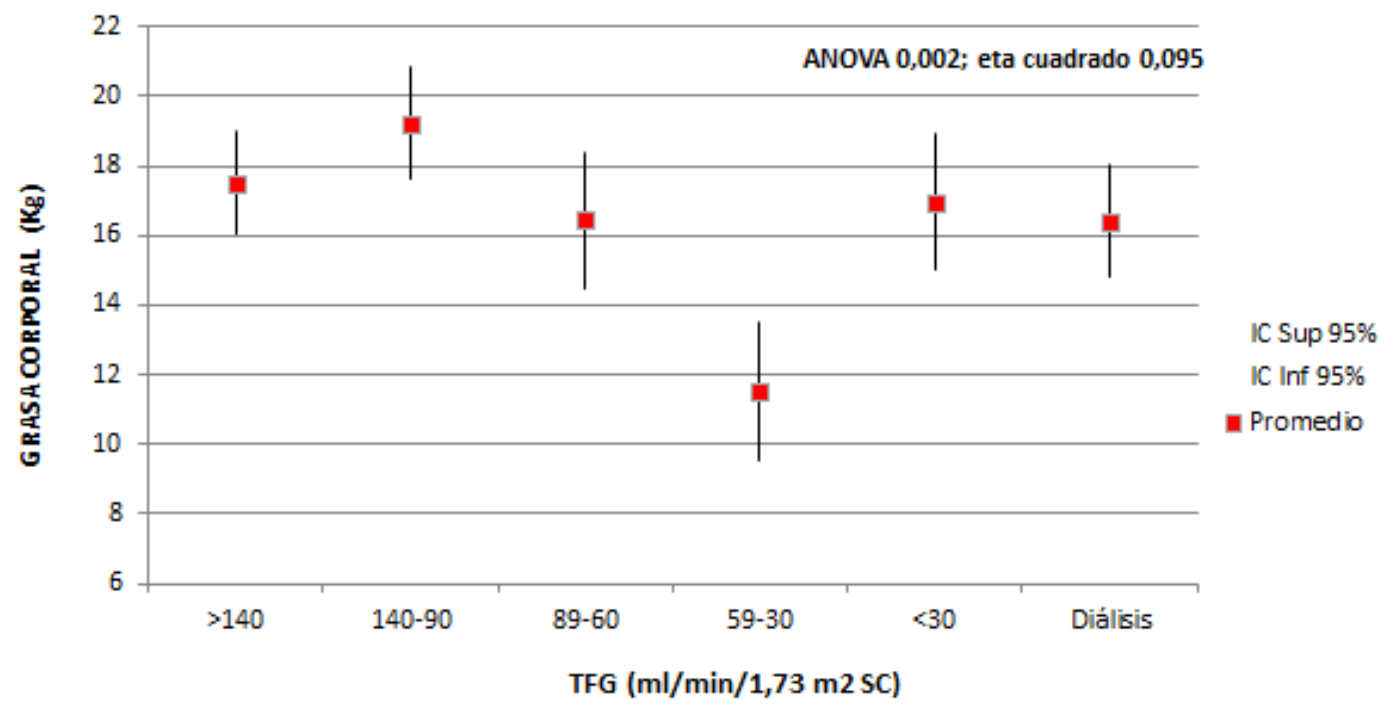

Gráfico 3. Relación entre la grasa corporal y el aclaramiento de creatinina categorizado de los sujetos estudiados.

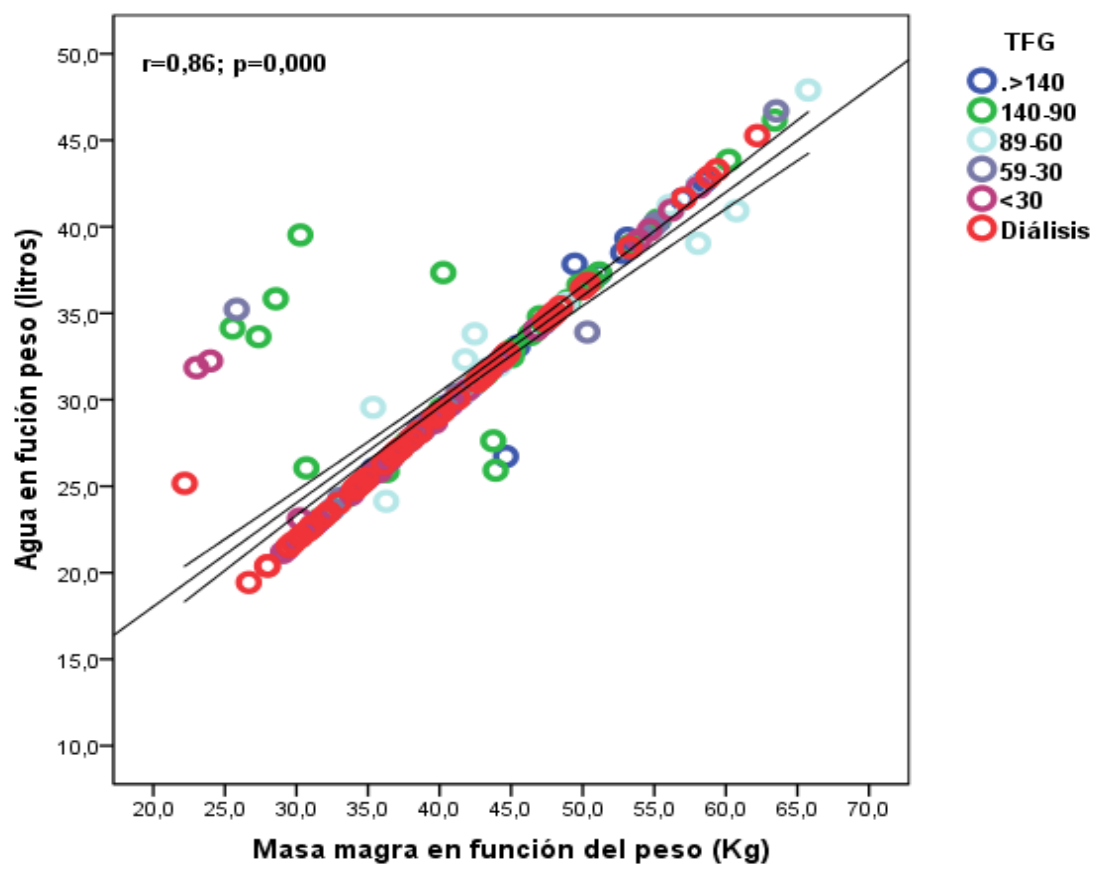

Gráfico 4. Correlación entre la masa magra y el agua corporal.

\section{DISCUSIÓN}

El estudio tuvo como objetivo principal describir los cambios del agua, grasa y masa magra corporal en el proceso de la salud a la enfermedad crónica avanzada, incluyendo personas que ya se encontraban en terapia de soporte renal de diálisis. Aun cuando el número de sujetos evaluados no fue igual en todos los grupos, fue suficientemente importante para permitirnos apreciar lo relevante de los cambios corporales que ocurren en el ser humano de nuestra sociedad entre la salud y la enfermedad renal crónica terminal, subrayando que, 
las categorías fueron normalizadas en función de la variable de confusión más relevante que puede existir al respecto, el sexo.

La edad permite notar que la disfunción renal en esta serie de casos es concordante con hechos ya conocidos, como el aumento de la incidencia de la enfermedad renal crónica en estados terminales con la edad (tabla 1 y 7) (4,5). La tabla 4 también permite apreciar otra variable relevante relacionada a la disfunción renal, la talla, pero esta variable no resultó significativa en el análisis lineal multivariado. La tabla 2 permite ver que no hubo ningún sujeto sano independiente de la edad que tuviera una depuración de creatinina inferior a los $60 \mathrm{ml} / \mathrm{min}$ (1). Mientras que tanto pacientes crónicos aun cuando estuvieran asintomáticos y antropométricamente parecieran sanos (tablas 2 y 3 ), pueden tener una TFG por debajo de $60 \mathrm{~m} / \mathrm{min}$.

La tabla 3 muestra que, la masa magra ajustada al peso de la persona, es la variable más relevante, cuyo eta cuadrado es muy fuerte, seguido por el agua corporal con quien mantiene una correlación lineal excelente en toda la extensión de la salud a la enfermedad renal avanzada como se aprecia en el gráfico 4. La grasa que también tiene una ANOVA estadísticamente significativo, a diferencia de la masa magra y el agua, tiene un nivel de efecto bastante inferior a estas variables que se refleja mejor en el gráfico 3 .

Estos datos infieren que si bien hay cambios en todas las estructuras corporales al disminuir la TFG, la masa magra y el agua son las variables relevantes en la reestructuración de la persona con disfunción renal en nuestra población respecto a los sanos. (6) Los gráficos 1 y 4 permiten apreciar la correlación antes descrita.

Puede verse que la caída de la masa magra ocurre como un proceso continuo, pero altamente notoria cuando el aclaramiento de creatinina está debajo de $30 \mathrm{ml} / \mathrm{min} / 1,73 \mathrm{~m}^{2} \mathrm{SC}$ (gráfico 1). Es decir, cuando ya el paciente está en el estadio 4 de la enfermedad renal crónica, las estructuras fundamentales para la vida se encuentran en procesos muy deteriorados $(7,8,9)$. En el gráfico 5 queda mucho más claro que cuando la creatinina sérica se encuentra por encima de los 2,5 $\mathrm{mg} / \mathrm{dl}$ este proceso es suficientemente grave. Es así que en un estudio de supervivencia realizado en España encuentran que la estructura corporal está asociada con la mortalidad en pacientes con enfermedad renal avanzada, y además que la baja masa magra es un factor de riesgo independiente para mortalidad (8).

En este punto es interesante volver a insistir que habiendo personas estudiadas con rangos avanzados de edad, los sujetos sanos pueden tener TFG menor de 90 $\mathrm{ml} / \mathrm{min} / 1,73 \mathrm{~m}^{2} \mathrm{SC}$, pero ninguno tiene valor inferior a $60 \mathrm{ml} / \mathrm{min}$ como ya se ha publicado anteriormente. Esto puede explicar algunas de las varianzas de los sujetos hasta depuraciones de creatinina mayores de $60 \mathrm{ml} /$ $\min / 1,73 \mathrm{~m}^{2} \mathrm{SC}$. En contraste, enfermos crónicos aún muy poco sintomáticos pueden tener TFG muy bajas, incluso hasta inferiores de $30 \mathrm{ml} / \mathrm{min}$. Los claramente sintomáticos ya tienen TFG menores de $30 \mathrm{ml} / \mathrm{min} \sin$ duda alguna (tabla 2).

Sintetizando los hallazgos, podríamos decir que las grandes estructuras corporales sufren todo un proceso de deterioro en dos variables fundamentales: el agua y la masa magra $(10,11,12)$. La grasa en este punto parecería ser la variable de interface que permite una vida bastante aceptable con seguridad hasta una TFG de $60 \mathrm{ml} / \mathrm{min}$. Debajo de este valor el proceso de transición se torna difícil para el enfermo y por debajo de $\operatorname{los} 30 \mathrm{ml} / \mathrm{min}$ parece impedir una calidad de vida aceptable y la persona sintomática (13). Es notorio que hay pocas diferencias en las estructuras corporales de las personas con TFG debajo de los $30 \mathrm{ml} / \mathrm{min}$ y el estar en terapia de soporte renal. Es probable que la menor edad observada en los pacientes en diálisis obedezca al hecho de que los pacientes de mayor edad no pueden sostener una vida biológica y fallecen antes de entrar a terapias de reemplazo renal.

Esta hipótesis se aúna a la observación de la correlación existente en la ingesta dietética de elementos fundamentales para la vida como proteínas de alto valor biológico y potasio puesto que su reserva biológica celular se encuentra ya bastante deteriorada (14).

El deterioro de la función renal está relacionado fundamentalmente con la edad, hecho ya muy bien reconocido en la literatura y que obedece, indiscutiblemente a que, este deterioro deviene de enfermedades crónicas altamente relevantes encima de los cincuenta años de la vida: la diabetes tipo 2 y la hipertensión arterial $(4,5)$. Sin embargo, el patrón de daño estructural es un proceso homogéneo que permite concluir que la atención del enfermo crónico independiente de su etiología, debe ser vista como una estructura integral y no compartimentada. En este sentido el quehacer médico debe estar estrechamente 
ligado al manejo nutricional, rehabilitación física, psicológica y social, enfocados en los aspectos fundamentales de mantener una estructura corporal adecuada en lo posible $(15,16)$. Esto no requiere de tecnología sofisticada y puede ser realizada en niveles de atención de la salud básicos con efectos positivos altamente eficientes.

\section{Declaración de financiamiento y de conflictos de intereses:}

El estudio fue financiado por los autores. Declaran no tener conflictos de intereses.

\section{Declaración de autoría:}

ARVS, JACZ, CUL: Participaron en el diseño del estudio, recolección de los datos, interpretación de los resultados y aprobación de la versión final a ser publicada.

\section{Correspondencia:}

Ana Rosa Vilela Sangay

Correo electrónico: ana.vilela@upch.pe

\section{REFERENCIAS BIBLIOGRÁFICAS}

1. Vilela AR, Cieza JA. Características de la estructura corporal, hábitos dietéticos y función renal a través de la vida, de personas saludables de clase media entre los 20 y 80 años de edad de Lima. Rev Med Hered. [Internet]. 2018 Oct [citado 10 junio de 2019]; 29(4): 217-225. Disponible en: http://www.scielo.org.pe/scielo.php?script= sci_arttext\&pid=S1018-130X2018000400003 \&lng=es. $\quad$ http://dx.doi.org/https://doi.org/10.20 453/rmh.v29i4.3446

2. Ku E, Kopple JD, Johansen $\mathrm{KL}$, McCulloch CE, Go AS, Xie D, Lin F, Hamm LL, He J, Kusek JW, Navaneethan SD, Ricardo AC, Rincon-Choles H, Smogorzewski M, Hsu CY. Longitudinal Weight Change during CKD Progression and Its Association With Subsequent Mortality. Am J Kidney Dis. 2018 May; 71(5):657-665. doi: 10.1053/j.ajkd.2017.09.015

3. Oliveira EA, Cheung WW, Toma KG, Mak RH. Muscle wasting in chronic kidney disease. Pediatr Nephrol. 2018 May; 33(5):789-798. doi: 10.1007/s00467-017-3684-6.

4. Kooman JP, van der Sande FM, Leunissen KM. Kidney disease and aging: A reciprocal relation. Exp Gerontol. 2017 Jan; 87(Pt B):156-
159. doi: 10.1016/j.exger.2016.02.003.

5. O'Sullivan ED, Hughes J, Ferenbach DA. Renal Aging: Causes and Consequences. J Am Soc Nephrol. 2017 Feb; 28(2):407-420. doi: 10.1681/ ASN.2015121308.

6. Caravaca F., Martínez del Viejo C., Villa J., Martínez Gallardo R., Ferreira F.. Estimación del estado de hidratación mediante bioimpedancia espectroscópica multifrecuencia en la enfermedad renal crónica avanzada. Nefrología (Madr.) [Internet]. 2011 [citado el 09 de julio de 2019]; 31( 5 ): 537-544.

7. Zhou Y, Hellberg M, Svensson P, Höglund P, Clyne N. Sarcopenia and relationships between muscle mass, measured glomerular filtration rate and physical function in patients with chronic kidney disease stages 3-5.Nephrol Dial Transplant. 2018 Feb 1; 33(2):342-348. doi: 10.1093/ndt/gfw466.

8. Vega A, Abad S, Macías N, et al. Low lean tissue mass is an independent risk factor for mortality in patients with stages 4 and 5 non-dialysis chronic kidney disease. Clin Kidney J. 2017; 10(2):170 175. doi:10.1093/ckj/sfw126.

9. Gracia-Iguacel Carolina, González-Parra Emilio, Pérez-Gómez M. Vanesa, Mahíllo Ignacio, Egido Jesús, Ortiz Alberto et al . Prevalencia del síndrome de desgaste proteico-energético y su asociación con mortalidad en pacientes en hemodiálisis en un centro en España. Nefrología (Madr.). 2013; 33(4):495505.

10. Schardong J, Marcolino MAZ, Plentz RDM. Muscle Atrophy in Chronic Kidney Disease.Adv Exp Med Biol. 2018; 1088:393-412. doi: 10.1007/978-98113-1435-3_18.

11. Lin TY, Peng CH, Hung SC, Tarng DC. Body composition is associated with clinical outcomes in patients with non-dialysis-dependent chronic kidney disease. Kidney Int.2018 Mar; 93(3):733740. doi: 10.1016/j.kint.2017.08.025.

12. Kim J, Kim S, Oh J, Lee Y, Noh J, Kim H, et al. Impact of sarcopenia on longterm mortality and cardiovascular events in patients undergoing hemodialysis. Korean J Intern Med. 2017. Nov; 10(39):1-9

13. Dong Y, Wang Z, Chen Z, et al. Comparison of visceral, body fat indices and anthropometric measures in relation to chronic kidney disease among Chinese adults from a large scale crosssectional study. BMC Nephrol. 2018; 19(1):40.

14. Barril G, Nogueira A, Ruperto López M, Castro Y, Sánchez-Tomero JA.Influence of dietary protein intake on body composition in chronic kidney disease patients in stages 3-5: A cross- 
sectional study. Nefrologia. 2018 Nov - Dec; 38(6):647-654. doi: 10.1016/j.nefro.2018.06.007.

15. Segall L, Mardare NG, Ungureanu S, Busuioc M, Nistor I, Enache R, Marian S, Covic A.Nutritional status evaluation and survival in haemodialysis patients in one centre from Romania. Nephrol Dial Transplant. 2009 Aug; 24(8):2536-40. doi: 10.1093/ndt/gfp110.
16. Otero González Alfonso, Iglesias Forneiro Alfonso, Camba Caride María Jesús, Pérez Melón Cristina, Borrajo Prol María Paz, Novoa Fernández Enrique et al. Supervivencia en hemodiálisis vs. diálisis peritoneal y por transferencia de técnica: experiencia en Ourense 1976-2012. Nefrología (Madr.) 2015; 35(6):562-566.

Recibido: $29 / 11 / 2019$

Aceptado: 30/03/2020 